\title{
The impact of COVID-19 pandemic on gastrointestinal diseases: a single-center cross-sectional study in central Greece
}

\author{
Apostolis Papaefthymiou ${ }^{\mathrm{a}, \mathrm{b}}$, Apostolos Koffas ${ }^{\mathrm{a}}$, Jannis Kountouras ${ }^{\mathrm{b}}$, Michael Doulberis ${ }^{\mathrm{b}, \mathrm{c}}$, Agoritsa Kaltsa ${ }^{\mathrm{a}}$, \\ Fotis Tsiopoulos ${ }^{\mathrm{a}}$, Grigorios Christodoulidis ${ }^{\mathrm{d}}$, Andreas Kapsoritakis ${ }^{\mathrm{a}}$, Spyros Potamianos ${ }^{\mathrm{a}}$ \\ University Hospital of Larissa, Thessaly, Greece; Ippokration Hospital, Aristotle University of Thessaloniki, Macedonia, \\ Greece; Medical University Department, Kantonsspital Aarau, Switzerland
}

\section{Abstract}

${ }^{a}$ Department of Gastroenterology, University Hospital of Larissa, Thessaly, Greece (Apostolis Papaefthymiou, Apostolos Koffas, Agoritsa Kaltsa, Fotis Tsiopoulos, Andreas Kapsoritakis, Spyros Potamianos); ${ }^{\mathrm{b}}$ Department of Internal Medicine, Second Medical Clinic, Ippokration Hospital, Aristotle University of Thessaloniki, Macedonia, Greece (Apostolis Papaefthymiou, Jannis Kountouras, Michael Doulberis); 'Division of Gastroenterology and Hepatology, Medical University Department, Kantonsspital Aarau, Switzerland (Michael Doulberis); dDepartment of Surgery, University Hospital of Larissa, Thessaly, Greece (Grigorios Christodoulidis)

\section{Conflict of Interest: None}

Correspondence to: Spyros Potamianos, Professor of Gastroenterology, Head of the department of Gastroenterology, University Hospital of Larissa, 16 Sklyrou str., Larissa, Thessaly, Greece,

e-mail: spotam@med.uth.gr

Received 30 August 2020; accepted 30 November 2020; published online 26 February 2021

DOI: https://doi.org/10.20524/aog.2021.0600

\section{Introduction}

COVID-19 pandemic, caused by SARS-CoV-2, has affected more than 87 million patients globally [1]. The current COVID-19 outbreak and the raised transmission, morbidity and mortality rates have led to the introduction of novel approaches in public health management and have induced social fundamental changes among populations [2]. The International Health organizations and Governments of all affected nations carry out urgent quarantine and hygiene procedures $[3,4]$. However, the varied, nonspecific clinical presentation and the high number of non-symptomatic virus carriers resulted in controversial outcomes [5-7].

Specifically, the emergence and rapid global spread of the deadly novel SARS-CoV-2 along with the absence of relative experience and literal data, motivated healthcare professionals and social scientists determining governmental policies, to 
adopt horizontal measures, thus triggering people's reservedness against the COVID-19 and the survival instinct [8]. Those conditions established widely restrictive policies and readjusted the role of healthcare systems, thus prioritizing the COVID-19 management, and probably underestimating the collateral impact from other diseases. Relevant reports indicated an increased all-cause mortality worldwide, probably reflecting the indirect burden of the pandemic and the respective quarantine [9-12]. In this regard, emerging data revealed a diminished admission rate or late admission to the hospital for pathologies irrelevant to the pandemic, due to patients' own fear, being more intense among the high-risk groups and patients with chronic illnesses [13], or their inability to visit a hospital [14-16]. For instance, if assumably $40 \%$ of people are not seeking care for acute coronary syndromes, and supposing $40 \%$ of those untreated people suffer a major complication or death, the effect of an ongoing COVID-19 pandemic on nonCOVID-related health issue of the population could be even more devastating than COVID-19 itself [17].

In Greece, the early lockdown and authorities' austerity during the first outbreak achieved a positive outcome in COVID-19 spread and relative deaths, thus constituting our country as a recognizable example of successful crisis management, with low healthcare expenditures among the Western societies. Governmental policy established a total lockdown and traffic limitations, lasting 42 days, discouraging any "unnecessary" transportation. Emerging data comparing this period to the respective previous year's one, underlined the absence of statistically significant increase of non-COVID-19 related deaths, based on Greek autopsy reports [18]. Nevertheless, the other side of the coin has not been fully investigated yet, owing to the absence of epidemiological comparative studies during the quarantine concerning nonCOVID-19-related hospital admissions. The aim of the current cross-sectional single-center study was to evaluate the potential impact of the lockdown period on the non-COVID-19 patients' outcome in a COVID-19 reference hospital department of gastroenterology.

\section{Patients and methods}

A single-center, cross-sectional study was conducted using the records of an internal electronic database of patients hospitalized in the Department of Gastroenterology of the General University Hospital of Larissa. This academic tertiary hospital offers its medical services in an area of approximately 1 million citizens [19]. During the COVID-19 pandemic, it served as a reference center for all relevant cases in the region. Datasets from 2 matched periods were retrieved, i.e., the lockdown period from March 23 to May 42020 and the respective previous year's timeframe (March 23 - May 4 2019). The study protocol conformed to the ethical guidelines of the last revision of Declaration of Helsinki and complied with Good Clinical Practice Guidelines [20,21]. The study was approved by the respective bioethics committee (Protocol Number: 48880) and patients' anonymity was ensured.
All adult patients ( $\geq 18$ years old), hospitalized in the gastroenterology department, after initial evaluation in the emergency department (ED), were eligible to participate in the study. Exclusion criteria comprised: a) scheduled/selective admissions and referrals for medical examinations, therapies or surveillance; and b) missing or incomplete data in the electronic database.

\section{Data collection and extraction}

Our own hospitalized patients during the aforementioned periods were recruited. Two groups were included according to the period of hospitalization, based on the lockdown. The eligibility of the included cases was evaluated by A.P. Patients' records were retrieved by A.P. and A.K. from AKTIS (v.1.6.8 for Windows, Aktis Computers Software ${ }^{\circledR}$, Greece), a clinical application which enables the storage and retrieval of multiple medical reports including history, clinical findings, laboratory and imaging examinations, endoscopic procedures and outcome information of all patients admitted. The following variables were evaluated: 1) sex; 2) age; 3) comorbidities, quantified according to the Charlson Comorbidity Index (CCI) [22]; 4) presenting symptom(s) upon admission; 5) definitive diagnosis; 6) therapeutic management (conservative, endoscopic treatment, or surgery); 7) duration of hospitalization; and 8) concluding outcome (positive, negative). Positive outcome was considered an uncomplicated course and discharge, whereas death and complications were merged as negative outcomes. An Excel file (Microsoft ${ }^{\circledR}$ Excel for Mac 2019, Microsoft Corporation, Redmond, WA, USA) was created and the variable values were extracted. All data have been stored in a secure server. In cases of conflict, a consensus was met by the intervention of a senior author (S.P.).

\section{Statistical analysis}

Data were presented as mean \pm standard deviation (SD) and percentages, for continuous and categorical variables, respectively. The comparisons of continuous and categorical variables were performed with the Mann-Whitney test and chi-square $\left(\chi^{2}\right)$ test (or the Fischer exact test), respectively. The normality of distribution of continuous variables was tested with the Kolmogorov-Smirnov test and Mann-Whitney test used for comparisons between groups. Binary logistic regression analysis was performed to investigate whether the lockdown period was independently associated with the hospitalization outcome. As additional independent variables, we considered parameters which could affect the patients' clinical course (comorbidities and diagnosis), as well as age and sex. Finally, sensitivity analysis was performed considering as cases with negative outcome only those who died, to investigate any potential impact on our results from cases with complicated but finally treated disease. Statistical analysis was performed with SPSS 21.0 for Macintosh (IBM Corp., Armonk, NY, USA). Significance was set at $\mathrm{P}<0.05$, 2-tailed. 


\section{Results}

Of the 727 hospitalized patients, 161 (22\%) fulfilled the inclusion criteria and were finally enrolled. Male to female ratio was almost 1:1 (82 men and 79 women) and mean age was 70.86 years (SD 18.61) without significant differences between the subgroups. Nearly two thirds of the admissions were due to gastrointestinal (GI) tract bleeding or biliary stone disease manifestations, $10.6 \%(n=17)$ of patients presented with a GI malignancy complication, $6.2 \%(n=10)$ presented with a flare of inflammatory bowel disease, and 6.8\% $(n=11)$ displayed GI tract infection, namely associated with Clostridium difficile. The vast majority $(85.1 \%, \mathrm{n}=137)$ of cases exhibited no complications, whereas 10 patients died, commonly due to complications relative to their underlying condition.

Fewer patients were hospitalized during the lockdown period (40\%) with melena and abdominal pain being the most commonly recognized symptoms for both compared timeframes $(\mathrm{P}=0.628)$. Non-variceal upper GI bleeding (UGIB) and gallstone complications were the most frequent diagnoses for both periods, whereas cases of variceal bleeding or acute pancreatitis were increased during the lockdown, though not statistically significant $(\mathrm{P}=0.693)$. Regarding comorbidities, the mean values of CCI between the subgroups did not differ significantly $(\mathrm{P}=0.553)$. The mainstay treatment approach was conservative (49.4\%) for both subgroups, though during the lockdown period a significantly higher percentage of patients required surgical intervention $(\mathrm{P}=0.006)$. Likewise, statistically significant difference $(\mathrm{P}=0.001)$ in the duration of in-patient accommodation was observed between the 2 subgroups. More specifically, during the lockdown, patients were hospitalized for $7.69 \pm 4.55$ days compared with $5.76 \pm 4.36$ days for their "non-lockdown" peers (Fig. 1). Table 1 illustrates the main differences between the 2 subgroups.

The potential difference of the final outcome (positive or negative) between the studied periods was estimated as a dependent variable in a binary regression analysis adjusted for potential confounders (Table 2). The lockdown period was positively associated with a negative outcome (odds ratio $[\mathrm{OR}]$ 5.21, 95\% confidence interval [CI] 1.66-16.34; $\mathrm{P}=0.005)$, especially concerning cases of GI malignancy and acute pancreatitis $(\mathrm{P}=0.045$ and 0.041 respectively). Sensitivity analysis (Table 3 ) included only patients who were deceased in the negative outcome subgroup and the significant relationship between outcome and quarantine was preserved $(\mathrm{P}=0.24)$. Additionally, the increasing CCI and the presence of GI cancer were independently positively associated with mortality, whereas female sex negatively (OR 0.073, 95\%CI 0.005-0.97; $\mathrm{P}=0.048)$.

\section{Discussion}

This cross-sectional study aimed to evaluate the impact of the lockdown period on the non-COVID-19 patients'

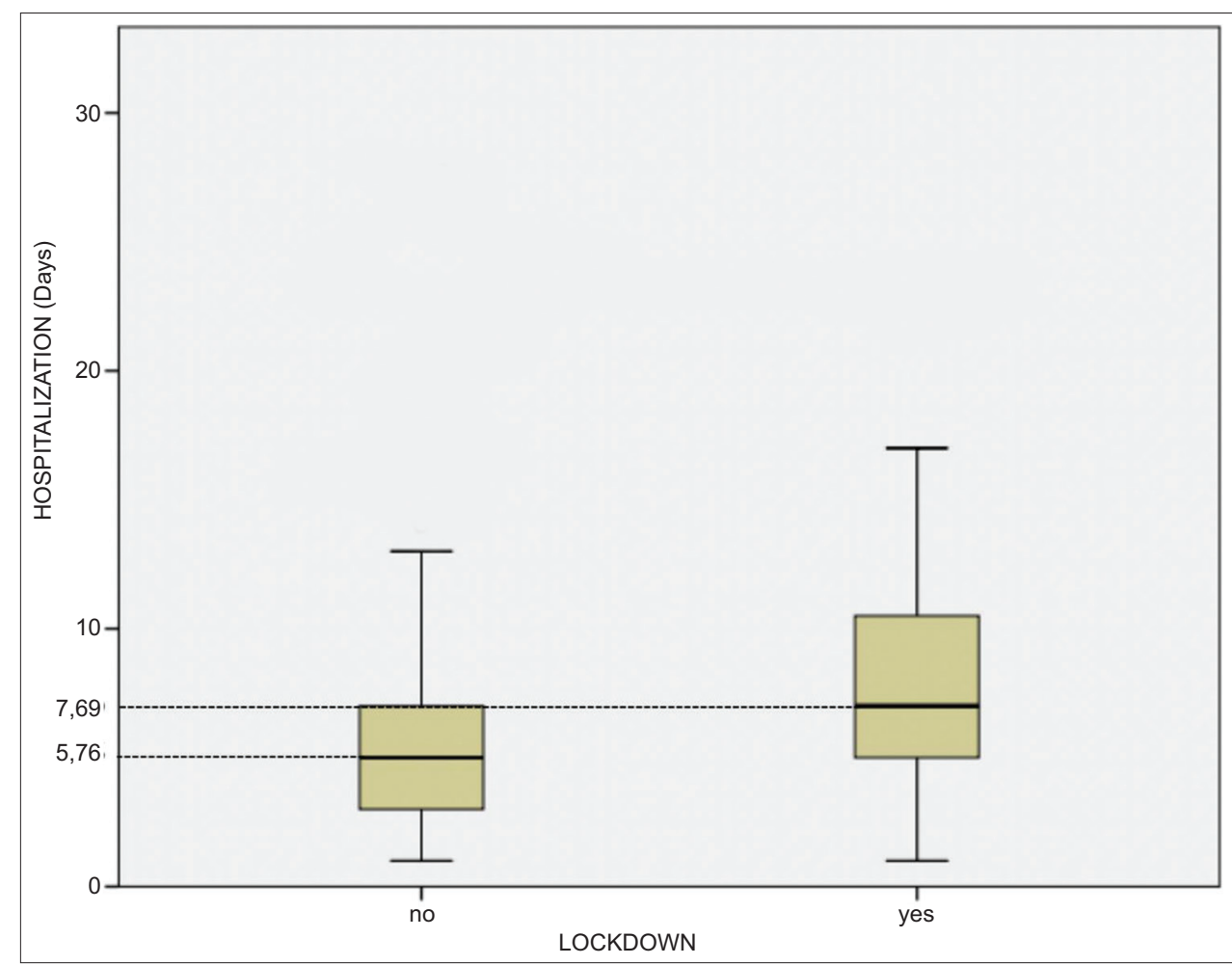

Figure 1 Box plot comparing the distribution of the hospitalization's duration between the lockdown period and the respective previous year's timeframe. The black dotted line interprets the median hospitalization, expressed in days, for every group. Statistical significance has been demonstrated between the 2 groups $(\mathrm{P}=0.001)$ 
4 A. Papaefthymiou et al

Table 1 Comparative data between the non-lockdown and lockdown groups

\begin{tabular}{|c|c|c|c|}
\hline Characteristics & Non-lockdown & Lockdown & P-value \\
\hline Male/Female [N(\%)] & $46 / 49(48,4 / 51,6)$ & $36 / 30(54.5 / 45.5)$ & 0.445 \\
\hline Mean age $\pm \mathrm{SD}$ (years) & $72.44 \pm 118.96$ & $68.59 \pm 18$ & \\
\hline $\mathrm{CCI} \pm \mathrm{SD}$ & $4.41 \pm 2.8$ & $4.14 \pm 2.67$ & 0.553 \\
\hline Symptom on admission $[\mathrm{N}(\%)]$ & & & 0.628 \\
\hline Melena & $19(20)$ & $22(33.3)$ & \\
\hline Hematochezia & $9(9.5)$ & $4(6.1)$ & \\
\hline Hematemesis & $4(4.2)$ & $2(3)$ & \\
\hline Fever/RUQ pain/jaundice ${ }^{* *}$ & $16(16.8)$ & $5(7.6)$ & \\
\hline Isolated jaundice & $4(4.2)$ & $3(4.5)$ & \\
\hline Abdominal pain & $31(32.6)$ & $21(31.8)$ & \\
\hline Vomiting & $2(2.1)$ & 0 & \\
\hline Isolated diarrhea & $6(6.3)$ & $6(9.1)$ & \\
\hline Diarrhea and fever/pain & $1(1.1)$ & $1(1.5)$ & \\
\hline Abnormal LFTs & $1(1.1)$ & $1(1.5)$ & \\
\hline Dysphagia & $2(2.1)$ & $1(1.5)$ & \\
\hline Diagnosis [N(\%)] & & & 0.693 \\
\hline Non-variceal UGIB & $21(22.1)$ & $15(22.7)$ & \\
\hline Gastrointestinal cancer & $3(3.2)$ & $2(3)$ & \\
\hline LGIB & $6(6.3)$ & $5(7.6)$ & \\
\hline Gallstone-related disease & $34(35.8)$ & $16(24.2)$ & \\
\hline Variceal bleeding & $1(1.1)$ & $4(6.1)$ & \\
\hline Liver abscess & $2(2.1)$ & $1(1.5)$ & \\
\hline Acute pancreatitis & $4(4.2)$ & $6(9.1)$ & \\
\hline Inflammatory bowel disease recurrence & $5(5.3)$ & $5(7.6)$ & \\
\hline Gastrointestinal infection & $8(8.4)$ & $3(4.5)$ & \\
\hline Sigmoid volvulus & 0 & $1(1.5)$ & \\
\hline Ischemic colitis & $2(2.1)$ & $2(3)$ & \\
\hline Pancreatobiliary cancer & $7(7.4)$ & $5(7.6)$ & \\
\hline Food impaction & $1(1.1)$ & $1(1.5)$ & \\
\hline Cirrhosis decompensation & $1(1.1)$ & 0 & \\
\hline Duration of hospitalization $\pm \mathrm{SD}$ (days) & $5.76 \pm 4.36$ & $7.69 \pm 4.55$ & 0.001 \\
\hline Treatment $[\mathrm{N}(\%)]$ & & & 0.013 \\
\hline Conservative & $46(48.4)$ & $32(48.5)$ & \\
\hline Endoscopic & $47(49.5)$ & $25(37.9)$ & \\
\hline Surgical & $2(2.1)$ & $9(13.6)$ & \\
\hline Outcome $[\mathrm{N}(\%)]$ & & & 0.006 \\
\hline Positive & $88(92.6)$ & $49(74.2)$ & \\
\hline Complication & $4(4.2)$ & $10(15.2)$ & \\
\hline Death & $3(3.2)$ & $7(10.6)$ & \\
\hline
\end{tabular}


Table 2 Independent associates of outcome (positive vs. negative) in binary logistic regression analysis

\begin{tabular}{|c|c|c|c|c|}
\hline Factors & Beta & Odds Ratios & $95 \% \mathrm{CI}$ & $\mathrm{P}$-value \\
\hline \multicolumn{5}{|l|}{ Sex } \\
\hline \multicolumn{5}{|l|}{ Male } \\
\hline Female & -0.328 & 0.72 & $0.24-2.17$ & 0.56 \\
\hline Age & -0.024 & 0.98 & $0.93-1.02$ & 0.3 \\
\hline CCI & 0.185 & 1.204 & $0.93-1.56$ & 0.162 \\
\hline \multicolumn{5}{|l|}{ Diagnosis } \\
\hline \multicolumn{5}{|l|}{ Non-variceal UGIB } \\
\hline Gastrointestinal cancer & 2.233 & 9.33 & $1.05-83.07$ & 0.045 \\
\hline LGIB & -1.099 & 0.33 & $0.03-3.3$ & 0.35 \\
\hline Gallstone-related disease & -1.35 & 0.26 & $0.06-1.15$ & 0.075 \\
\hline Variceal bleeding & -0.87 & 0.42 & $0.04-4.95$ & 0.49 \\
\hline Liver abscess & 0.774 & 2.17 & $0.12-39.84$ & 0.6 \\
\hline Acute pancreatitis & 1.752 & 5.77 & $1.08-3-.93$ & 0.041 \\
\hline Inflammatory bowel disease recurrence & -20.292 & 0 & & 0.99 \\
\hline Gastrointestinal infection & -19.863 & 0 & & 0.99 \\
\hline Sigmoid volvulus & -20.813 & 0 & & $>0.99$ \\
\hline Ischemic colitis & -20.053 & 0 & & 0.99 \\
\hline Pancreatobiliary cancer & -1.336 & 0.26 & & 0.241 \\
\hline Food impaction & -20.273 & 0 & & 0.99 \\
\hline Cirrhosis decompensation & -20.734 & 0 & & $>0.99$ \\
\hline \multicolumn{5}{|l|}{ Lockdown } \\
\hline \multicolumn{5}{|l|}{ No } \\
\hline Yes & 1.65 & 5.21 & $1.66-16.34$ & 0.005 \\
\hline
\end{tabular}

outcome in Thessaly, an area of central Greece. Our findings clearly demonstrated an association between the COVID-19 pandemic lockdown and the hospitalization outcome of patients compared to the respective previous year's subgroup, matched for sex, mean age, comorbidities, and cause of admission. Indeed, during the recent quarantine period the inpatients were 5.2 times more likely to experience a negative outcome and the mortality raised to $10.6 \%$ compared to the previous year's $3.2 \%$ (Fig. 2). These data were also supported by the statistically significant increase in the mean hospitalization time and the increased requirement for surgical interventions.

The aforementioned deflection in cases' outcomes during the quarantine could be catalysed by the delayed admission in the ED from symptoms' onset. Emerging data indicated that during the COVID-19 outbreak significant delays have been reported in diagnosing and managing life-threatening conditions [23]. In this respect, patients with chronic diseases, such as malignancies, are vulnerable, and the equilibrium between fear of immunosuppression, with the threat of COVID-19, and a compromised outcome owing to either

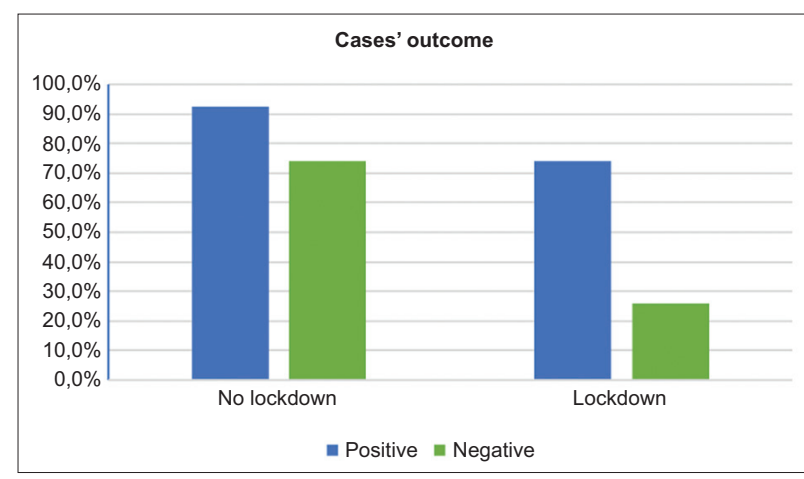

Figure 2 Graphical interpretation of the hospitalization outcomes based on the "lockdown" variable. More cases (odds ratio 5.21, 95\% confidence interval 1.66-16.34; $\mathrm{P}=0.005$ ) experienced a negative outcome during the COVID-19 outbreak in Greece compared to the respective period in 2019

delays in treatment or the usage of suboptimal alternatives is a problem of great concern occurring daily throughout the world. For instance, the patients' delayed decision to undergo scheduled endoscopic procedures and the relevant official 
Table 3 Sensitivity analysis regarding outcome (positive vs death) in binary logistic regression analysis

\begin{tabular}{|c|c|c|c|c|}
\hline Factors & Beta & Odds Ratios & $95 \% \mathrm{CI}$ & P-value \\
\hline \multicolumn{5}{|l|}{ Sex } \\
\hline \multicolumn{5}{|l|}{ Male } \\
\hline Female & -2.624 & 0.073 & $0.005-0.97$ & 0.048 \\
\hline Age & 0.038 & 1.039 & $0.934-1.16$ & 0.48 \\
\hline CCI & 0.545 & 0.545 & $0.545-2.48$ & 0.545 \\
\hline \multicolumn{5}{|l|}{ Diagnosis } \\
\hline \multicolumn{5}{|l|}{ Non-variceal UGIB } \\
\hline Gastrointestinal cancer & 4.057 & 57.776 & $1.275-2167.8$ & 0.037 \\
\hline LGIB & -18.123 & 0 & 0 & $>0.99$ \\
\hline Gallstone-related disease & 0.857 & 2.357 & $0.233-23.84$ & 0.468 \\
\hline Variceal bleeding & 2.279 & 9.771 & $0.195-489.83$ & 0.254 \\
\hline Liver abscess & -19.718 & 0 & 0 & $>0.99$ \\
\hline Acute pancreatitis & 1.44 & 4.222 & $0.168-105.85$ & 0.381 \\
\hline Inflammatory bowel disease recurrence & -16.24 & 0 & 0 & $>0.99$ \\
\hline Gastrointestinal infection & -17.68 & 0 & 0 & $>0.99$ \\
\hline Sigmoid volvulus & -15.177 & 0 & 0 & $>0.99$ \\
\hline Ischemic colitis & -18.816 & 0 & 0 & $>0.99$ \\
\hline Pancreatobiliary cancer & 0.077 & 1.08 & $0.062-18.71$ & 0.958 \\
\hline Food impaction & -18.358 & 0 & 0 & $>0.99$ \\
\hline Cirrhosis decompensation & -22.022 & 0 & 0 & $>0.99$ \\
\hline \multicolumn{5}{|l|}{ Lockdown } \\
\hline \multicolumn{5}{|l|}{ No } \\
\hline Yes & 2.386 & 10.867 & $1.371-86.14$ & 0.024 \\
\hline
\end{tabular}

guidelines, which suggested against elective or surveillance endoscopies, during the coronavirus outbreak, appears to grow the GI cancer-related deaths [24,25]; and postponing potentially curative cancer surgery can also lead to severe complications such as bowel obstruction [26].

During the lockdown, acute severe biliary pancreatitis was a significant confounder burdening our patients' clinical course, thereby necessitating surgical intervention. This outcome seems to be comparable with Mallick's et al [27] findings, that recognized late patient presentation as an independent risk factor of increased morbidity and mortality associated with acute pancreatitis. Likewise, late endoscopy appears to increase mean hospitalization time and mortality in patients with UGIB [28]. In our study, an increased rate of neglected cases was recorded during the lockdown and the percentages of patients admitted during the pre- and COVID-19 era were similar, whereas the prevalence of UGIB cases was diminished in Austria during the lockdown [29].

During the era of the COVID-19 pandemic, healthcare systems across the globe adopted restrictive policies and implemented structural reforms to minimize the viral spread and the consequent morbidity and mortality associated with it. It is important to comment, however, that the impact of quarantine and limitation of social contacts should not be underestimated or drop to the bottom of the governmental agenda because they may lead to high human negative emotions and economic costs [30]. Likewise, pandemics, like coronavirus, brought tremendous epidemiological as well as psychological consequences (i.e., anxiety, depression, insomnia, trauma, anger, psychosis, panic and boredom) similar to other pandemics in the past [30].

In Greece, the respective restrictive policy in the interim of the first outbreak resulted in a primary reduced COVID-19 transmission rate and lower complication and death rates, compared to other European countries. During the quarantine, any outdoor movement, except for emergencies, was discouraged and legal sanctions were imposed to offenders. Likewise, media campaigns and daily public updates were promoted to underline the necessity of staying at home and social distancing. Consequently, the cumulative implementation of these interventions, alongside the instilled 
anxiety about social interaction, led to restrictive movement of the population [8]. Moreover, mental health professionals recognized an increased incidence of the mentioned anxiety, negative emotional spirals, lower stress threshold, desperation, panic and fear [31,32]. Taking this into consideration, the rather increased prevalence of COVID-19 cases in our region, alongside the fact that our hospital was a COVID-19 reference center, has probably acted as inhibitory factor for early presentation and admission.

Respective reports from other specialties raised identical concerns, underlying the alarming indirect shortening of life-expectancy in COVID-19 irrelevant diseases during the lockdown [9-12,16,33]. In Greece, a recent study, performed by the medical group which managed the crisis and based on forensic medical reports, suggested similar mortality by non-COVID-19 causes during the lockdown and previous year's respective period [18]. More specifically, deaths due to sudden/unexpected reasons or myocardial infraction were increased during the lockdown period, though without obtained significant level. The authors concluded that information derived from forensic autopsies is an invaluable epidemiological tool, and given that in Greece autopsy is not necessary for all cases, the abovementioned sample could underestimate the real incidence of deaths during the lockdown period, especially concerning inpatients with established diagnosis before death. With this regard, the jeopardy of conditions with proven high mortality and disability potential, such as cardio- and cerebro-vascular diseases, has been downgraded during the pandemic even in well-organized healthcare systems, thus leading to a suspicious decrease in ED admissions for stroke and acute coronary syndromes $[14,33]$. Moreover, in countries that had to tackle with a high influx of COVID-19 patients, delays in medical interventions have been observed, likely resulting from the updated ED protocols and redistribution of human resources to manage COVID-19 cases [33].

This study has certain limitations. Initially, the observational design cannot prove an etiological relationship and data from other departments and/or centers including larger samples would be useful to achieve more interpretable results. Additionally, the comparison between the lockdown period and the respective previous year's timeframe seems arbitrary and a selection bias has to be acknowledged, albeit the matched parameters for both groups, thus providing relatively safe results.

In conclusion, the negative outcomes of hospitalized GI patients have been raised during the lockdown period, probably due to their delayed presentation, admission and compromised resources. The unprecedented scale of the pandemic has brought the governments, healthcare professionals and scientists around the world under tremendous pressure to devise control strategies and develop novel prevention measures. To avoid a further "double-sword" hit of relapsing outbreaks, balanced and well-designed approach considerations are warranted based on the recent and evolving experience of COVID-19 pandemic.

\section{Summary Box}

\section{What is already known:}

- The current COVID-19 pandemic has triggered horizontal and austere public health measures to limit its spread

- Healthcare systems prioritized the management of COVID-19, probably underestimating the collateral damage by non-COVID-19-related conditions

- Greek Authorities imposed an immediate and dynamic restrictive policy to inhibit the COVID-19 spread

\section{What the new findings are:}

- The duration of hospitalization during the lockdown period has been significantly increased for gastrointestinal (GI) disease patients

- The lockdown period was associated with worse clinical outcomes and a higher rate of complicationrelated surgery for hospitalized GI disease patients

- The lockdown period, GI malignancies and comorbidities were associated with increased mortality compared to the non-lockdown period

\section{References}

1. WHO. Coronavirus disease (COVID-19) pandemic. 2020. Available from: https://www.who.int/emergencies/diseases/novelcoronavirus-2019 [Accessed 7 January 2021].

2. Hens N, Vranck P, Molenberghs G. The COVID-19 epidemic, its mortality, and the role of non-pharmaceutical interventions. Eur Hear J Acute Cardiovasc Care. 2020;9:204-208.

3. Sohrabi C, Alsafi Z, O'Neill N, et al. World Health Organization declares global emergency: a review of the 2019 novel coronavirus (COVID-19). Int J Surg 2020;76:71-76.

4. Müller O, Neuhann F, Razum O. Epidemiologie und Kontrollmaßnahmen bei COVID-19. DMW - Dtsch Medizinische Wochenschrift 2020;145:670-674.

5. Gahide G, Frandon J, Vendrell J-F. COVID-19 patients presenting with afebrile acute abdominal pain. Clin Med (Northfield Il) 2020;20:e4-e6.

6. Boukhris M, Hillani A, Moroni F, et al. Cardiovascular implications of the COVID-19 pandemic: a global perspective. Can J Cardiol 2020;36:1068-1080.

7. Harris C, Carson G, Baillie JK, Horby P, Nair H. An evidence-based framework for priority clinical research questions for COVID-19. J Glob Health 2020;10:011001.

8. Harper CA, Satchell LP, Fido D, Latzman RD. Functional fear predicts public health compliance in the COVID-19 pandemic. Int J Ment Health Addict 2020;1-14.

9. Noche CD, Njajou O, Etoa FX. No Association between CagA- and VacA-positive strains of Helicobacter pylori and primary open-angle glaucoma: a case-control study. Ophthalmol Eye Dis 2016;8:1-4.

10. Weinberger DM, Chen J, Cohen T, et al. Estimation of excess 
deaths associated with the COVID-19 pandemic in the United States, March to May 2020. JAMA Intern Med 2020;180:1336-1344.

11. Piccininni M, Rohmann JL, Foresti L, Lurani C, Kurth T. Use of all cause mortality to quantify the consequences of covid-19 in Nembro, Lombardy: descriptive study. BMJ 2020;369:m1835.

12. Mannucci E, Nreu B, Monami M. Factors associated with increased all-cause mortality during the COVID-19 pandemic in Italy. Int $J$ Infect Dis 2020;98:121-124.

13. Satici B, Gocet-Tekin E, Deniz ME, Satici SA. Adaptation of the fear of COVID-19 scale: its association with psychological distress and life satisfaction in Turkey. Int J Ment Health Addict 2020 May 8 [Online ahead of print]. doi: 10.1007/s11469-020-00294-0

14. Naccarato M, Scali I, Olivo S, et al. Has COVID-19 played an unexpected "stroke" on the chain of survival? J Neurol Sci 2020;414:116889.

15. Viganò $M$, Voza $A$, Harari $S$, et al. Clinical management of nonrespiratory diseases in the COVID-19 pandemic: what have we done and what needs to be done? Telemed e-Health 2020;26:12061208.

16. Marijon E, Karam N, Jost D, et al. Out-of-hospital cardiac arrest during the COVID-19 pandemic in Paris, France: a populationbased, observational study. Lancet Public Heal 2020;5:E437-E443.

17. Metzler B, Siostrzonek P, Binder RK, Bauer A, Reinstadler SJ. Decline of acute coronary syndrome admissions in Austria since the outbreak of COVID-19: the pandemic response causes cardiac collateral damage. Eur Heart J 2020;41:1852-1853.

18. Sakelliadis EI, Katsos KD, Zouzia EI, Spiliopoulou CA, Tsiodras S. Impact of Covid-19 lockdown on characteristics of autopsy cases in Greece. Comparison between 2019 and 2020. Forensic Sci Int 2020;313:110365.

19. Hellenic Statistical Authority. 2011 Population and Housing Census Fertility Statistics.Available from: https://www. statistics.gr/documents/20181/1215267/A1602_SAM03_ DT_DC_00_2011_05_F_EN.pdf/beaac915-b865-490a-abfebfce5fb812dd [Accessed 11 November 2020].

20. European Medicines Agency (EMA). Guideline Good Clinical Practice E6(R2) 2018;6:1-68.

21. World Medical Association. Declaration of Helsinki, Ethical Principles for Scientific Requirements and Research Protocols. Bull
World Health Organ 2013;79:373.

22. Charlson ME, Pompei P, Ales KL, MacKenzie CR. A new method of classifying prognostic comorbidity in longitudinal studies: Development and validation. J Chronic Dis 1987;40:373-383.

23. Ho JS, Tambyah PA, Ho AF, Chan MY, Sia C-H. Effect of coronavirus infection on the human heart: A scoping review. Eur J Prev Cardiol 2020;27:1136-1148.

24. Armellini E, Repici A, Alvisi C, et al. Analysis of patients attitude to undergo urgent endoscopic procedures during COVID-19 outbreak in Italy. Dig Liver Dis 2020;52:695-699.

25. Gralnek IM, Hassan C, Beilenhoff U, et al. ESGE and ESGENA position statement on gastrointestinal endoscopy and the COVID-19 pandemic. Endoscopy 2020;52:483-490.

26. Finley C, Prashad A, Camuso N, et al. Guidance for management of cancer surgery during the COVID-19 pandemic. Can J Surg 2020;63:S2-S4.

27. Mallick B, Dhaka N, Sharma V, et al. Impact of timing of presentation of acute pancreatitis to a tertiary care centre on the outcome. Pancreatology 2019;19:143-148.

28. Jeong N, Kim KS, Jung YS, Kim T, Shin SM. Delayed endoscopy is associated with increased mortality in upper gastrointestinal hemorrhage. Am J Emerg Med 2019;37:277-280.

29. Schmiderer A, Schwaighofer H, Niederreiter L, et al. Decline in acute upper gastrointestinal bleeding during Covid-19 pandemic after lockdown in Austria. Endoscopy 2020;52:1036-1038.

30. Cerbara L, Ciancimino G, Crescimbene M, Longa FLA. A nationwide survey on emotional and psychological impacts of COVID-19 social distancing. Eur Rev Med Pharmacol Sci 2020;24:7155-7163.

31. Hiremath P, Suhas Kowshik CS, Manjunath M, Shettar M. COVID 19: Impact of lock-down on mental health and tips to overcome. Asian J Psychiatr 2020;51:102088.

32. Kaparounaki CK, Patsali ME, Mousa D-PV, Papadopoulou EVK, Papadopoulou KKK, Fountoulakis KN. University students' mental health amidst the COVID-19 quarantine in Greece. Psychiatry Res 2020;290:113111.

33. Bersano A, Kraemer M, Touzé E, et al. Stroke care during the Covid-19 pandemic: Experience from three large European countries. Eur J Neurol 2020;27:1794-1800. 